and for $k \gg \lambda$ the behaviour resembles that of a charge (this depends on the assumptions made regarding the internal field). This behaviour is illustrated in Fig. 1.

The peculiar form of the above expression lends itsclf readily to at least qualitative experimental test, and may help to determine the field of a neutron. The calculations are being extended to other models and to the consideration of nuclear collisions.

Cavendish Laboratory, H. S. W. Massey. Cambridge.

1 Natrke, 129, $312 ; 1932$

2 Vide C'arlson and oppenheimer, Phys, Rev, 38, 1787; 1931.

3 Bakerian Lecture, Proe. Roy. Soc., A, 97, 374; 1920.

\section{Radioactive Disintegration and Nuclear Spin}

IT is well known that in radioactive disintegration, an a-particle escaping from the nucleus has to cross a potential barrier formed at great distances by Coulomb repulsion forees and at small distances by attractive forces of yet unknown nature. Wave mechanics permits us to calculate the transparency of such a barrier and to obtain the formula connecting the decay constant $\lambda$, the velocity $v$ of the ejected $a$-particle, the charge number $Z$ of the disintegrating element, and the radius $r_{0}$ of the internal nuclear region. ${ }^{1}$

Using the experimental values of $Z, v$ and $\lambda$, it is possible to calculate the radii of different radioactive nuclei which are shown in Fig. 1. For the thorium

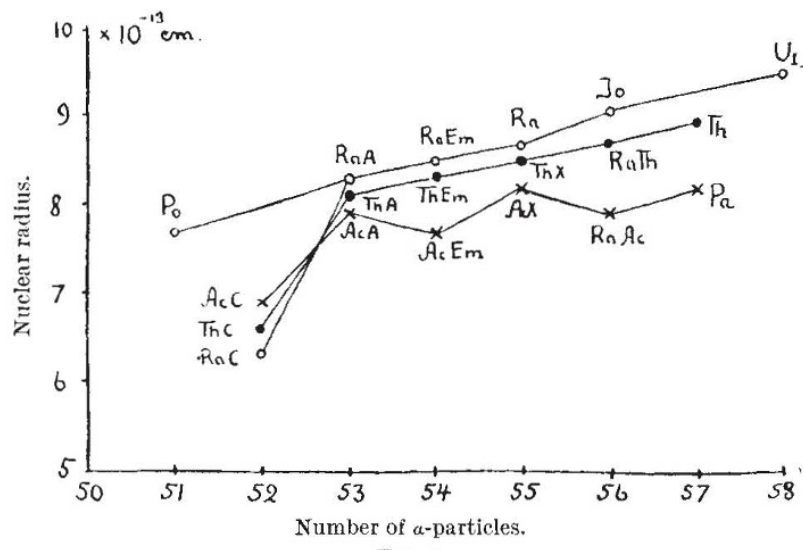

Fig. 1.

and uranium families the values of the nuclear radii vary rather regularly with increasing atomic weight, whereas for the actinium family a number of irregularities is present. This may be either because the radii of this family really do vary irregularly, or because all the factors are not taken in account by the calculations.

In the formula by means of which the values of radii shown in Fig. I have been calculated, the angular momentum $i$ of an escaping $a$-particle was supposed to be zero. The presence of angular momentum increases the height of the potential barrier (the centrifugal force added to the Coulomb forces) and gives smaller values of the decay constant for the given $r_{0}$, or, the other way round, the neglect of this momentum leads to too small values of the radius, which is just the case for some members of actinium family. On the other hand, if nuclear spins of different elements which have been investigated are examined, it is seen that the nuclei with even atomic weights (to which the thorium and uranium families belong) usually have no spin at all, whereas for those with odd atomic weights (for example, the actinium family) the nuclear momenta can take rather different values (from $\frac{1}{2}$ up to $\left.\frac{9}{2}\right)$.

If the nuclei of the elements which are disintegrating and their products have different spins, the ejected a-particles must have angular momentum. The formula for the decay constant can be, in this case, written in the form :

$$
\begin{aligned}
& \lambda_{j}=\frac{\pi h}{2 m r_{0}^{2}} \cdot e^{-\frac{4 \pi e^{2}}{h}} \frac{(Z-2)}{v}+\frac{8 e \sqrt{m}}{h} \sqrt{(Z-2) \cdot} \sqrt{ } r_{0}(1-\sigma) \\
& \sigma=\frac{h^{2}}{2 m} \frac{j(j+1)}{r_{0}{ }^{2}}: \frac{2(Z-2) e^{2}}{r_{0}}=0.002 j(j+1),
\end{aligned}
$$

where $h$ denotes (Planck's constant) $/ 2 \pi$.

Using this formula we can, for example, estimate the correct value of the atomic radius of actinium emanation by ascribing to the $a$-particle escaping from this nucleus an angular momentum of about three units, which means that the difference between the nuclear spins of actinium emanation and actinium- $A$ is about $\frac{{ }_{2}}{2}$.

Such calculations, although uncertain, can give some idea of the spins of radioactive nuclei. Considerations of the same kind can also be applied to the decay processes when escaping $a$-particles leave the nucleus in an excited state, ${ }^{2}$ although in these cases the estimations must be still more uncertain owing to the increase of the nuclear radius by excitation.

Club of Scientists,

G. Ga mow.

Leningrad, Feb. 8.

1 G. Gamow, "Constitution of Atomic Nuclei and Radioactivity," Oxford. 1931.

\&. Gamow, NaTURE, Sept. 13, 1930.

\section{Molecular Structure of Polysaccharides}

IN the issue of Nature of March 5, Prof. W. N. Haworth announces that he has isolated tetramethyl glucose by hydrolysis of a fully methylated cellulose, and, on the basis of this result, he makes certain suggestions regarding the probable dimensions of the cellulose chain. In addition, he states that he is undertaking a similar research on other polysaccharides, and the letter closes with a claim for reservation.

It ought not to be necessary to point out that this laboratory, in which the methylation process was developed and first applied to all types of carbohydrates, has been engaged continuously for more than twelve years on the general problem now included in Prof. Haworth's reservation. The results reported by him, and others of a similar nature, have been known to us for a considerable time, but we have good grounds for the opinion that the interpretation of these results is beset with so many complications that early publication is inadvisable. At regular intervals during the past four or five years, however, the results have been communicated to the expert referees of the Carnegie Trust, which has, in part, financed the work.

In the circumstances which have emerged it appears to be desirable, in the interests of my collaborators, to synopsise, so far as this is expedient, some of the results already obtained by us.

Cellulose.-When cellulose is methylated on a large scale and the product subjected to graded hydrolysis, only $2: 3: 6$-trimethyl glucose is liberated in the first instance, the $2: 3: 4: 6$-tetramethyl glucose being derived from the more resistant fraction of the methylated cellulose. It is still an open question if this result can be interpreted as a proof that cellulose possesses a long-chain structure, or if the amount of tetramethyl glucose formed can be used as the basis of calculating the length of the cellulose chain as a whole. This work has been conducted, with numerous variations, on cellulose derived from 